\title{
The IRMA code for unique classification of medical images
}

\author{
Thomas M. Lehmann ${ }^{*}$, Henning Schubert ${ }^{\mathrm{a}}$, Daniel Keysers ${ }^{\mathrm{b}}$, \\ Michael Kohnen ${ }^{\mathrm{a}}$, Berthold B. Wein ${ }^{\mathrm{a}}$ \\ Department of Medical Informatics \\ ${ }^{a}$ Department of Diagnostic Radiology \\ ${ }^{\mathrm{b}}$ Chair of Computer Science VI \\ Aachen University of Technology (RWTH), Aachen, Germany
}

\begin{abstract}
Modern communication standards such as Digital Imaging and Communication in Medicine (DICOM) include nonimage data for a standardized description of study, patient, or technical parameters. However, these tags are rather roughly structured, ambiguous, and often optional. In this paper, we present a mono-hierarchical multi-axial classification code for medical images and emphasize its advantages for content-based image retrieval in medical applications (IRMA). Our so called IRMA coding system consists of four axes with three to four positions, each in $\{0, \ldots 9, \mathrm{a}, \ldots, \mathrm{z}\}$, where " 0 " denotes "unspecified" to determine the end of a path along an axis. In particular, the technical code $(\mathrm{T})$ describes the imaging modality; the directional code (D) models body orientations; the anatomical code (A) refers to the body region examined; and the biological code (B) describes the biological system examined. Hence, the entire code results in a character string of not more than 13 characters (IRMA: TTTT - DDD - AAA - BBB). The code can be easily extended by introducing characters in certain code positions, e.g., if new modalities are introduced. In contrast to other approaches, mixtures of one- and two-literal code positions are avoided which simplifies automatic code processing. Furthermore, the IRMA code obviates ambiguities resulting from overlapping code elements within the same level. Although this code was originally designed to be used in the IRMA project, other use of it is welcome.
\end{abstract}

Keywords: Standardized Nomenclature, Digital Imaging and Communication in Medicine (DICOM), Picture Archiving and Communication Systems (PACS), Content-Based Image Retrieval (CBIR), Image Classification Code

\section{INTRODUCTION}

Modern communication standards include non-image data for a standardized description of study, patient, body region examined, and technical parameters related to the imaging modality in use. In order to provide comprehensive, detailed coverage for multi-specialty biomedical imaging, the College of American Pathologists (CAP), secretariat of the Systematized Nomenclature of Human and Veterinary Medicine (SNOMED), has entered into partnership with the Digital Imaging and Communications in Medicine (DICOM) Standards Committee and other professional organizations to develop a nomenclature that is needed for diagnostic imaging applications [1]. The SNOMED DICOM microglossary was developed to provide context-dependent value sets for DICOM coded-entry data elements, and semantic content specifications for reports and other structures composed of multiple data elements [2].

Although the capability of storing explicitly-labeled coded descriptors in DICOM images and reports improves the potential for selective retrieval of images and related information, the controlled terminology within the DICOM tables has been found to be insufficiently detailed for order entry systems [3] or even invalid, if values are set automatically by the system [4]. Concerning the needs for content-based image retrieval in medical applications (IRMA), a detailed coding scheme is required to describe (a) the imaging modality including technical parameters, (b) the orientation of the image with respect to the body, (c) the body region examined, and (d) the biological system investigated $[5,6]$.

\footnotetext{
*1ehmann@computer.org; phone +49 241 80-88793; fax +49 241 80-82426; http://www.irma-project.org; Department of Medical Informatics, Aachen University of Technology, Pauwelsstr. 30, D - 52057 Aachen, Germany.
} 
In this paper, we present a mono-hierarchical (i.e., every son-node is connected to only one parent-node) multi-axial (i.e., more tan one semantic axes) classification code for medical images [7] in English and emphasize its advantages for image retrieval in comparison to existing proposals supplementary to the DICOM standard.

\section{METHODS}

Within the IRMA system [5], categorization is the first of seven successive analyzing steps extracting content information from medical images. The categorization aims to establish intelligent processing strategies adapted to the current image under investigation. Therefore, valid relations between code and sub-code elements are "is-a" and "partof" only. Consequently, the code must be strictly hierarchical in order to support semantic queries on a database. Furthermore, causality is important for grouping of processing strategies. Therefore, a mono-hierarchical scheme is required, where each sub-code element is connected to only one code element. Since categorization of medical images must cover all aspects influencing the image content and structure, a multi-axial scheme was designed. This scheme, which has been presented previously in German [7] is now available in English, as well.

\section{RESULTS}

The IRMA coding system consists of four axes with three to four positions, each in $\{0, \ldots 9, \mathrm{a}, \ldots, \mathrm{z}\}$, where "0" denotes "unspecified" to determine the end of a path along an axis:

- T (technical): image modality

- D (directional): body orientation

- A (anatomical): body region examined

- B (biological): biological system examined

This allows a short and unambiguous notation (IRMA: TTTT - DDD - AAA - BBB), where T, D, A, and B denotes a coding or sub-coding digit of the technical, directional, anatomical, and biological axis, respectively. In addition, this notation avoids mixtures of one- and two-literal code positions.

\subsection{Technical code for imaging modality}

The T-code describes within a maximum of four positions the technical method. It starts with the physical source of image acquisition (e.g.: 1 "x-ray", 2 "ultrasound", 3 "magnetic resonance imaging", 4 "optical imaging", ...) showing more details in the modality position (e.g.: 11 "plain film projection radiography", 12 "fluoroscopy", 13 "angiography", 14 "computed tomography", ...). A third digit specifies the technique (e.g.: 111 "digital", 112 "analog", 113 "stereometry", 114 "stereography", ...), and the fourth position of the T-code assesses sub-techniques (e.g.: 1111 "tomography", 1112 "high energy", 1113 "low energy", 1114 "parallel beam", ...). A complete listing of the IRMA T-code is given in Appendix A. Note that the non-radiological part of this code (4 "nuclear medicine", 5 "optical imaging", 6 "biophysical procedures", 7 "others", 8 "secondary digitization") are not modeled completely.

\subsection{Directional code for imaging orientation}

This three-digit part of the IRMA-code incorporates a two-step orientation description starting with the common orientation (e.g.: 1 "coronal", 2 "sagittal", 3 "transversal", 4 "other") and giving a more detailed specification in the second position (e.g.: 11 "posteroanterior (PA)", 12 "anteroposterior (AP) "). Note that it is important to distinguish APand PA-directions since organs and bone structures might differ in scale, for instance, supposing plain X-ray chest imaging. Independent from the relative orientation of body region and imaging system, functional orientation tasks of the examination can also be described (e.g.: 111 "inspiration", 112 "expiration", 113, "valsalva", 114 "phonation", ...). A complete listing of the IRMA D-code is given in Appendix B.

\subsection{Anatomical code for body region examined}

The IRMA-code supports complete coding of the anatomical region. In total, nine major regions are defined (e.g.: 1 "total body", 2 "head/scull", 3 "spine", 4 "upper extremity", ...). The major region is followed by up to two 
hierarchical sub-codes (e.g.: 3 "spine", 31 "cervical spine", 311 "dens"). A complete listing of the IRMA A-code is given in Appendix C.

\subsection{Biological code for biological system examined}

The B-code determines the organ system that is imaged. This axis is necessary because the body region examined insufficiently describes content and structure of images. For example, fluoroscopy of the abdominal region may access the vascular or the gastrointestinal system depending on the way the contrast agent is administered, which results in different image textures. On the top-level of this three digit IRMA-code, ten organ systems are specified (e.g.: 1 "cerebrospinal system", 2 "cardiovascular system", 3 "respiratory system", 4 "gastrointestinal system", ...) each of which having up to three positions to exactly identify the organ in question (e.g.: 1 "cerebrospinal system", 11 "central nervous system", 111 "mesencephalon"). A complete listing of the IRMA B-code is given in Appendix D.

\subsection{Examples of image coding}

A web-based interface has been established for coding of radiographs [8]. All images are converted to readable icons of about $200 \times 200$ pixels and labeled with the appropriate code by two professional readers, i.e. board certified radiologists. This allowed the assessment of the quality of computerized image categorization and also to create a gold standard. The labeling tool is based on an relational database addressed with standard query language (postgreSQL 7.1.3), an interface to a web-server (apache 1.3.26 with built-in php 4.2.2 interpreter) and on the client-site on a standard internet browser (mozilla 1.1). The appropriate code is entered and remarks of the readers are collected. The code can be entered directly into the appropriate field, if known by heart, or composed - making extensive use of javascripting in the HTML-file to enable modifying pop-up menus according to the decision made at higher levels. This resulted in a stepwise refinement of the code. The radiological report is available simultaneously to judge for specific comments. Thereafter, coded images are transferred anonymously from the routine application into the IRMA research case database [8].

Figure 1 gives two examples of unambiguous image classification using the IRMA-code. The image on the left is coded: "x-ray, projection radiography, analog, high energy - sagittal, left lateral decubitus, inspiration - chest, lung respiratory sytem, lung. The image on the right is coded: x-ray, fluoroscopy, analog - coronad, ap, supine - abdomen, upper abdomen, middle - gastrointestinal system, stomach".

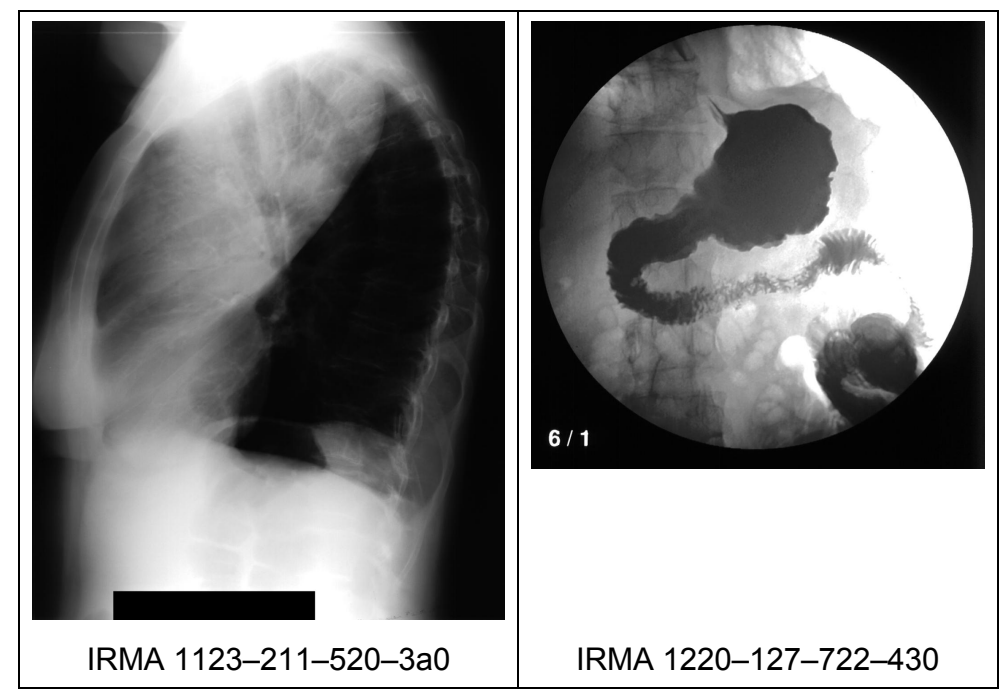

Figure 1: IRMA-coded chest and abdomen radiograph. 


\section{DISCUSSION}

A basic requirement for coding medical images according to processing within a system for content-based retrieval is the mono-hierarchical structure of the code. Although several nomenclatures exist, these are neither causal, hierarchical, complete, nor unambiguous. For instance, valid instances for the DICOM tag "body part examined" are "skull", "cspine", "tspine", "lspine", "sspine", "coccyx", "chest", "clavicle", "breast", "abdomen", "pelvis", "hip", "shoulder", "elbow", "knee", "ankle", "hand", "foot", "extremity", "head", "heart", "neck", "arm", "jaw", and "special" [9]. Note the different grade of differentiation (e.g., "cspine", "tspine", "Ispine", "sspine" vs. "skull"), the incompleteness (e.g., "arm" but not "leg"), and the ambiguities (e.g., "extremity" vs. "arm" or "hand").

The MeSH thesaurus is a poly-hierarchical structure where entities can be reached on different paths [10]. For instance, the IRMA T-code 1111 "dual energy digital plain x-ray" corresponds to the $2002 \mathrm{MeSH}$ codes E01.370.350.700.700.700, E01.370.350.760.700, and E01.370.350.600.350.700.700 for "radiography, dual energy scanned projection". Furthermore, the MeSH thesaurus is not sufficiently detailed in all of the IRMA axis, especially for the T-axis.

Although it was demonstrated that SNOMED [11] is the most complete reference terminology in existence today for the clinical environment [12], its incompleteness with respect to technical usage for image retrieval reveals a major disadvantage of SNOMED when compared to the IRMA code. For instance, the SNOMED DICOM microglossary offers "breast" but not "mammary gland" [3].

In summary, the IRMA coding scheme is closest related to the JJ1017 code [3]. Likewise the JJ1017 approach, the IRMA code enables image classification with respect to technical, directional, anatomical, and biological criterions. In contrast to JJ1017, IRMA is more detailed, offers four instead of three separated axes for the four parameters described above, and is strictly mono-hierarchical in each axis. In addition, ambiguities of JJ1017 are avoided. For instance, JJ1017 offers "chest", "chest/abdomen", and "abdomen" on the first level of its "large region code" and hence, it is not suitable for content-based image retrieval in medical applications.

Based on its structure, the IRMA code can easily be extended (adding new characters to a certain code position) and refined. For instance, the IRMA B-code 411 "oral cavity" for the biological system imaged can be further specified if required for other usage than content-based image retrieval, e.g., 4110 "unspecified", 4111 "tongue" 4112 "floor of mouth", 4113 "hard palate", 4114 "soft palate", 4115 "cheek".

So far, the coding support was easy to use in about 6,000 single images, the loading time for the small images (about 200 pixels either direction) was short, the support of the javascripts in case of unknown combinations of the coding scheme was helpful. It turned out that rarely (less than $0.1 \%$ ) the regional code is too limited to describe the body parts depicted on a radiograph. This is especially true for long extremity overviews. The extremity codes allows only a very general or a highly specific localization of a combination of two nearby regions [8].

\section{CONCLUSION}

A mono-hierarchical multi-axial code scheme is presented that enables a unique classification of medical images. The entire code results in a character string of not more than 13 characters. The code can be easily extended by introducing characters in certain code positions, e.g., if new modalities are introduced. In contrast to other approaches, mixtures of one- and two-literal code positions are avoided which simplifies automatic code processing. Furthermore, the IRMA code steers clear of ambiguities resulting from overlapping code elements within the same level. Although this code was originally designed to be used in the IRMA project, other use is welcome.

\section{ACKNOWLEDGEMENT}

This work was performed within the image retrieval in medical applications (IRMA) project, which is supported by the German Research Community (Deutsche Forschungsgemeinschaft, DFG) grants Le 1108/4 and Le 1108/6. 


\section{REFERENCES}

1. Bidgood WD, Korman LY, Golichowski AM, Hildebrand PL, Mori AR, Bray BB, Brown NJG, Spackman KA, Dove SB, Schoeffler K: Controlled terminology for clinically-relevant indexing and selective retrieval of biomedical images. International Journal on Digital Libraries 1997; 3(1): 278-287.

2. Bidgood WD: The SNOMED DICOM Microglossary - Controlled terminology resource for data interchange in biomedical imaging. Methods of Information in Medicine 1998; 37(4-5): 404-414.

3. Kimura M, Kuranishi M, Sukenobu Y, Watanabe H, Nakajima T, Morimura S, Kabata S: JJ1017 image examination order codes - standardized codes supplementary to DICOM for image modality, region, and direction. Proceedings SPIE 2002; 4685: 307-315.

4. Güld MO, Kohnen M, Keysers D, Schubert H, Wein BB, Bredno J, Lehmann TM: Quality of DICOM header information for image categorization. Proceedings SPIE 2002; 4685: 280-287.

5. Lehmann TM, Wein B, Dahmen J, Bredno J, Vogelsang F, Kohnen M: Content-based image retrieval in medical applications: A novel multi-step approach. Proceedings SPIE 2000; 3972: 312-320.

6. Tagare HD, Jaffe CC, Duncan J: Medical image databases - A content-based retrieval approach. Journal of the American Medical Informatics Association JAMIA 1997; 4(3): 184-198.

7. Lehmann TM, Wein BB, Keysers D, Kohnen M, Schubert H: A monohierarchical multiaxial classification code for medical images in content-based retrieval. Proceedings IEEE International Symposium on Biomedical Imaging, Washington, DC, July 7-10, 2002; 313-316.

8. Wein B, Lehmann TM, Keysers D, Schubert H, Kohnen M: Detailed image classification code for image retrieval of medical images (IRMA). In: Lemke HU, Vannier MW, Inamura K, Farman AG, Doi K, Reiber JHC: CARS 2002 - Computer Assisted Radiology and Surgery. Proceedings of the 16th International Congress and Exhibition Paris, Springer-Verlag, Berlin, 2002; 513-517.

9. National Electrical Manufacturers Association (NEMA) ed.: Digital Imaging and Communications in Medicine (DICOM) - Part 3, PS 3.3-2001. NEMA publishing, Rosslyn, VA, USA, 2001. (http://medical.nema.org/dicom/2001/01 03PU.PDF)

10. National Library of Medicine (NLM) ed: 2002 Medical Subject Headings (MeSH). National Technical Information Service (NTIS), Spingfield, VA, USA, 2002. (http://nlm.nih.gov/mesh)

11. Campbell JR, Carpenter P, Sneiderman C, Cohn S, Chute CG, Warren J. Phase II evaluation of clinical coding schemes - Completeness, taxonomy, mapping, definitions, and clarity. Journal of the American Medical Informatics Association JAMIA 1997; 4: 238-251.

12. Côté RA (ed): SNOMED - Systematized Nomenclature of Medicine. The College of American Pathologists, Northfield, IL, USA, 1982. (http://www.snomed.org/)

\section{APPENDIX}

\section{A Technical code for imaging modality}

Note that the non-radiological part of this code (4 "nuclear medicine", 5 "optical imaging", 6 "biophysical procedures", 7 "others", 8 "secondary digitization") are not modeled completely.

$0 \quad$ unspecified

1 x-ray

10 unspecified

11 plain radiography

110 unspecified

111 digital

1110 unspecified

1111 overview image
1112 tomography

1113 high beam energy

1114 low beam energy

1115 far view projection

1116 1:1 projection

1117 dual energy

analog

1120 unspecified

1121 overview image

1122 tomography

1123 high beam energy

1124 low beam energy

1125 far view projection

$1126 \quad 1: 1$ projection

113 x-ray stereometry

114 x-ray stereography 
115 tomosynthesis

116 xeroradiography

12 fluoroscopy

120 unspecified

121 digital

122 analog

13 angiography

130 unspecified

131 digital

1310 unspecified

1311 subtraction (DSA)

1312 rotation

angiography

1313 pathfinder

technique

132 analog

1320 unspecified

1321 photo subtraction

14 computed tomography (CT)

140 unspecified

141 conventional CT

1410 unspecified

1411 low dose

1412 high resolution

1413 bolus tracking

142 quantitative CT

1420 unspecified

1421 dual energy

15 DEXA (quantitative radiography)

150 unspecified

151 digital

1510 unspecified

1511 dual energy

16 radiotherapy

$\begin{array}{ll}160 & \text { unspecified } \\ 161 & \text { digital } \\ 162 & \text { analog }\end{array}$

2

sonography

20 unspecified

21 A-mode

210 unspecified

$221 \quad 3.5 \mathrm{MHz}$

$212 \quad 5.0 \mathrm{MHz}$

$213 \quad 7.5 \mathrm{MHz}$

$214 \quad 10 \mathrm{MHz}$

215 variable frequency

22 B-mode

220 unspecified

221 sector scan

2210 unspecified

$2211 \quad 3.5 \mathrm{MHz}$

$2212 \quad 5.0 \mathrm{MHz}$

$2213 \quad 7.5 \mathrm{MHz}$

$2214 \quad 10 \mathrm{MHz}$
222

2215 variable frequency

linear scan

2220 unspecified

$2221 \quad 3.5 \mathrm{MHz}$

$2222 \quad 5.0 \mathrm{MHz}$

$2223 \quad 7.5 \mathrm{MHz}$

$2224 \quad 10 \mathrm{MHz}$

2225 variable frequency

23

M-mode

230 unspecified

$231 \quad 3.5 \mathrm{MHz}$

$232 \quad 5.0 \mathrm{MHz}$

$233 \quad 7.5 \mathrm{MHz}$

$23410 \mathrm{MHz}$

235 variable frequency

24 compound mode

240 unspecified

$241 \quad 3.5 \mathrm{MHz}$

$242 \quad 5.0 \mathrm{MHz}$

$243 \quad 7.5 \mathrm{MHz}$

$24410 \mathrm{MHz}$

245 variable frequency

25 SieScape mode

250 unspecified

$251 \quad 3.5 \mathrm{MHz}$

$252 \quad 5.0 \mathrm{MHz}$

$253 \quad 7.5 \mathrm{MHz}$

$254 \quad 10 \mathrm{MHz}$

255 variable frequency

26 duplex mode

260 unspecified

$261 \quad 3.5 \mathrm{MHz}$

$262 \quad 5.0 \mathrm{MHz}$

$263 \quad 7.5 \mathrm{MHz}$

$26410 \mathrm{MHz}$

265 variable frequency

$27 \quad$ Doppler mode

270 unspecified

$271 \quad 3.5 \mathrm{MHz}$

$272 \quad 5.0 \mathrm{MHz}$

$273 \quad 7.5 \mathrm{MHz}$

$274 \quad 10 \mathrm{MHz}$

275 variable frequency

$28 \quad 3 \mathrm{D}$ imaging

280 unspecified

$281 \quad 3.5 \mathrm{MHz}$

$282 \quad 5.0 \mathrm{MHz}$

$283 \quad 7.5 \mathrm{MHz}$

$28410 \mathrm{MHz}$

285 variable frequency

3

magnetic resonance measurements

30 unspecified

31 magnetic resonance imaging (MRI) 310 unspecified 
T1-weigthed

3110 unspecified

3111 endo coil

3112 body

3113 body array

3114 head coil

3115 neck coil

3116 spine coil

3117 loop coil

3118 shoulder coil

3119 knee coil

311a hand / small FOV

311b mamma coil

311c flexible coil, large

311d flexible coil, small

311e endorectal coil

$311 \mathrm{f}$ wrap around coil

312 T2-weighted

3120 unspecified

3121 endo coil

3122 body

3123 body array

3124 head coil

3125 neck coil

3126 spine coil

3127 loop coil

3128 shoulder coil

3129 knee coil

312a hand / small FOV

312b mamma coil

312c flexible coil, large

312d flexible coil, small

312e endorectal coil

312f wrap around coil

313 proton-weighted

3130 unspecified

3131 endo coil

3132 body

3133 body array

3134 head coil

3135 neck coil

3136 spine coil

3137 loop coil

3138 shoulder coil

3139 knee coil

313a hand / small FOV

313b mamma coil

313c flexible coil, large

313d flexible coil, small

313e endorectal coil

313f wrap around coil

314 fat suppression

3140 unspecified

3141 endo coil
3142 body

3143 body array

3144 head coil

3145 neck coil

3146 spine coil

3147 loop coil

3148 shoulder coil

3149 knee coil

314a hand / small FOV

314b mamma coil

314c flexible coil, large

314 d flexible coil, small

314e endorectal coil

314f wrap around coil

315

Cine-Lolo

3150 unspecified

3151 endo coil

3152 body

3153 body array

3154 head coil

3155 neck coil

3156 spine coil

3157 loop coil

3158 shoulder coil

3159 knee coil

315a hand / small FOV

315b mamma coil

315c flexible coil, large

$315 \mathrm{~d}$ flexible coil, small

$315 \mathrm{e}$ endorectal coil

$315 f \quad$ wrap around coil

316 inversion recovery

3160 unspecified

3161 endo coil

3162 body

3163 body array

3164 head coil

3165 neck coil

3166 spine coil

3167 loop coil

3168 shoulder coil

3169 knee coil

316a hand / small FOV

316b mamma coil

316c flexible coil, large

316d flexible coil, small

316e endorectal coil

$316 \mathrm{f}$ wrap around coil

317 FLARE

3170 unspecified

3171 endo coil

3172 body

3173 body array

3174 head coil 


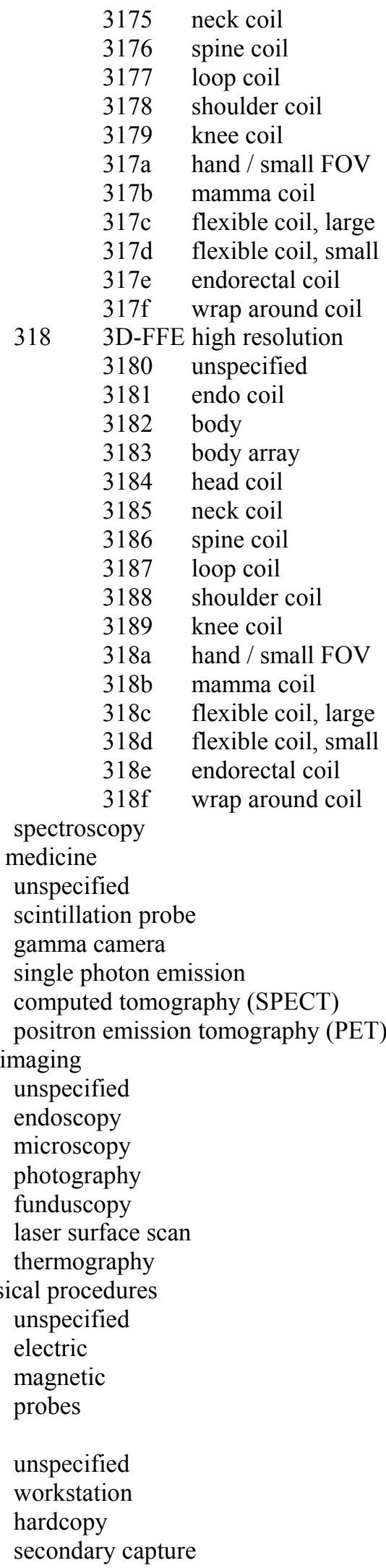

74

documentation

740 unspecified

741 value

742 curve local

743 curve temporal

744 area complex (3D)

745 picture

746 local series

747 temporal series

748 complex series (4D)

749 cine documentation

74a fluoroscopy

$74 \mathrm{~b}$ volume (data set)

$74 \mathrm{c} \quad$ volume temporal (data set)

8 secondary digitalization

80 unspecified

$81 \quad$ laser sampling

$82 \quad$ LCD sampling

83 video digitizing

\section{B Directional code for imaging orientation}

0 unspecified

1 coronal

\begin{tabular}{|c|c|c|}
\hline 10 & \multicolumn{2}{|c|}{ unspecified } \\
\hline 1 & \multicolumn{2}{|c|}{ posteroanterior (PA) } \\
\hline & 110 & unspecified \\
\hline & 111 & inspiration \\
\hline & 112 & expiration \\
\hline & 113 & valsalva \\
\hline & 114 & phonation \\
\hline & 115 & upright \\
\hline & 116 & sitting \\
\hline & 117 & supine \\
\hline & 118 & prone \\
\hline & 119 & lateral decubitus \\
\hline & $11 \mathrm{a}$ & flexion, left \\
\hline & $11 b$ & flexion, right \\
\hline & $11 \mathrm{c}$ & swallow \\
\hline & $11 d$ & micturition \\
\hline & $11 \mathrm{e}$ & bending \\
\hline \multirow[t]{14}{*}{12} & \multicolumn{2}{|c|}{ anteroposterior (AP, coronal) } \\
\hline & 120 & unspecified \\
\hline & 121 & inspiration \\
\hline & 122 & expiration \\
\hline & 123 & valsalva \\
\hline & 124 & phonation \\
\hline & 125 & upright \\
\hline & 126 & sitting \\
\hline & 127 & supine \\
\hline & 128 & prone \\
\hline & 129 & lateral decubitus \\
\hline & $12 \mathrm{a}$ & flexion, left \\
\hline & $12 b$ & flexion, right \\
\hline & $12 \mathrm{c}$ & swallow \\
\hline
\end{tabular}

Proc. of SPIE Vol. 5033 


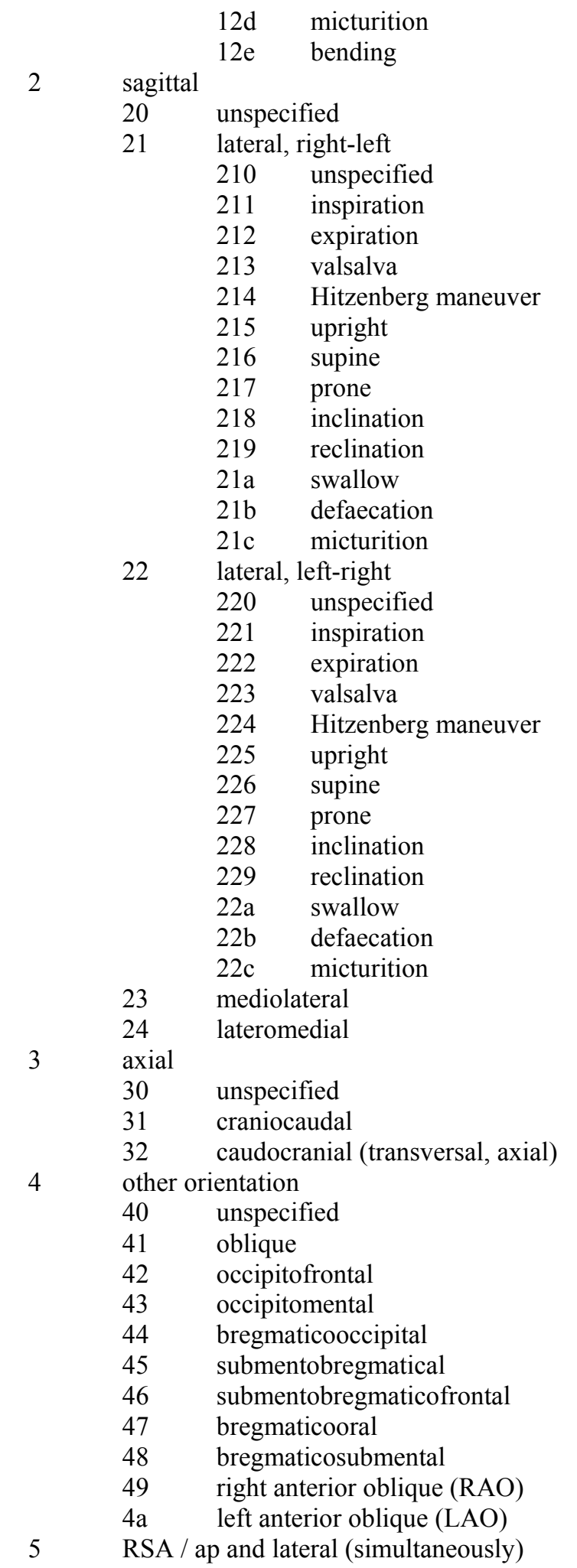

\section{Anatomical code for body region examined}

$0 \quad$ unspecified
1

whole body

$\begin{array}{ll}10 & \text { unspecified } \\ 11 & \text { torso } \\ 12 & \text { extremities }\end{array}$

2 cranium

20 unspecified

21 facial cranium

210 unspecified

211 forehead

212 eye area

213 nose area

214 maxilla

215 mandible

216 temporo mandibular area

22 cranial base

220 unspecified

221 petrous bone

222 sella

23 neuro cranium

230 unspecified

231 frontal area

232 parietal area

233 occipital area

3

spine

30 unspecified

31 cervical spine

310 unspecified

311 dens

312 axis

313 upper cervical spine

314 lower cervical spine

32 thoracic spine

320 unspecified

321 cervico-thoracic conjunction

322 upper thoracic spine

323 middle thoracic spine

324 lower thoracic spine

33 lumbar spine

330 unspecified

331 thoraco-lumbar conjunction

332 upper lumbar spine

333 lower lumbar spine

34 sacral bone

340 unspecified

341 lumbo-sacral conjunction

35 coccygeal bone

4 upper extremity / arm

$\begin{array}{lll}40 & \text { unspecified } \\ 41 & \text { hand } & \\ & 410 & \text { unspecified } \\ & 411 & \text { finger } \\ & 412 & \text { middle hand } \\ & 413 & \text { carpal bones } \\ 42 & & \text { radio carpal joint }\end{array}$


forearm

430 unspecified

431 distal forearm

432 proximal forearm

44 elbow

45 upper arm

450 unspecified

451 distal upper arm

452 proximal upper arm

46 shoulder

460 unspecified

461 scapula

462 humero-scapular joint

463 acromio-scapula joint

5 chest

50 unspecified

51 bones

510 unspecified

511 clavicle

512 sternoclavicle region

513 sternum

514 upper ribs

515 lower ribs

$52 \quad$ lung

520 unspecified

521 upper lobe

522 middle lobe

523 lower lobe

53 hilum

54 mediastinum

540 unspecified

541 anterior mediastinum

542 middle mediastinum

543 posterior mediastinum

55 heart

56 diaphragm

6 breast (mamma)

7 abdomen

70 unspecified

71 upper abdomen

710 unspecified

711 upper right quadrant

712 upper middle quadrant

713 upper left quadrant

72 middle abdomen

720 unspecified

721 middle right abdomen

722 peri navel region

723 middle left quadrant

73 lower abdomen

730 unspecified

731 lower right quadrant

732 lower middle quadrant

733 lower left quadrant
8

pelvis

80 unspecified

81 sarcral bone

810 unspecified

811 iliosacral-junction

82 iliac bone

83 pubic bone

830 unspecified

$831 \quad$ symphysis

84 small pelvis

840 unspecified

841 sacral bone

842 ischial bone

9 lower extremity / leg

90 unspecified

91 foot

910 unspecified

911 toe

912 middle foot

913 tarsal bones

92 ankle joint

93 lower leg

930 unspecified

931 distal lower leg

932 proximal lower leg

94 knee

940 unspecified

941 patella

95 upper leg

950 unspecified

951 distal upper leg

952 proximal upper leg

96 hip

D Biological code for system examined

0 unspecified

1 cerebrospinal system

10 unspecified

11 central nervous system

110 unspecified

111 metencephalon, cerebrum

112 mesencephalon,

diencephalon

113 neurohypophysis, posterior pituitary gland

114 cerebellum

115 pons

116 medulla oblongata

117 spinal cord, cervical

118 spinal cord, thoracic

119 spinal cord, lumbal

11a spinal cord, sacral

(cauda equina)

12 sense organs 


\begin{tabular}{|c|c|c|}
\hline \multicolumn{3}{|r|}{ unspecified } \\
\hline & 121 & vestibular sense \\
\hline & 122 & hearing sense \\
\hline & 123 & gustatory sense \\
\hline & 124 & tactile sense \\
\hline & 125 & vision sense \\
\hline & 126 & olfactory sense \\
\hline \multirow[t]{9}{*}{13} & vege & ve nervous system \\
\hline & 130 & unspecified \\
\hline & 131 & sympathic chain \\
\hline & 132 & glomus jugulare \\
\hline & 133 & glomus caroticum \\
\hline & 134 & ganglion stellatum \\
\hline & 135 & ganglion coeliacum \\
\hline & 136 & organ of Zuckerkandl \\
\hline & 137 & adrenal medulla \\
\hline \multicolumn{3}{|c|}{ cardiovascular system } \\
\hline \multirow{10}{*}{$\begin{array}{l}20 \\
21\end{array}$} & unsp & fied \\
\hline & hear & \\
\hline & 210 & unspecified \\
\hline & 211 & pericardium \\
\hline & 212 & atrium \\
\hline & 213 & mitral valve \\
\hline & 214 & tricuspidal valve \\
\hline & 215 & ventricle \\
\hline & 216 & aortic valve \\
\hline & 217 & pulmonary valve \\
\hline \multirow[t]{13}{*}{22} & arter & \\
\hline & 220 & unspecified \\
\hline & 221 & ascending aorta \\
\hline & 222 & aortic arche \\
\hline & 223 & brachiocephalic trunk \\
\hline & 224 & common carotic artery \\
\hline & 225 & $\begin{array}{l}\text { botalli ligament \& } \\
\text { ductus arteriosus }\end{array}$ \\
\hline & 226 & descending aorta \\
\hline & 227 & bronchial trunk \\
\hline & 228 & abdominal aorta \\
\hline & 229 & celiac trunk \\
\hline & $22 \mathrm{a}$ & common iliac artery \\
\hline & $22 b$ & other arteries \\
\hline \multirow[t]{8}{*}{23} & vein & \\
\hline & 230 & unspecified \\
\hline & 231 & anonymus vein \\
\hline & 232 & brachiocephalic vein \\
\hline & 233 & superior vena cava \\
\hline & 234 & inferior vena cava \\
\hline & 235 & common iliac vein \\
\hline & 236 & other veins \\
\hline \multicolumn{3}{|c|}{ respiratory system } \\
\hline 30 & \multicolumn{2}{|c|}{ unspecified } \\
\hline 31 & \multicolumn{2}{|c|}{ nose } \\
\hline 32 & \multicolumn{2}{|c|}{ sinuses } \\
\hline 33 & \multicolumn{2}{|c|}{ nasopharynx \& epipharynx } \\
\hline
\end{tabular}

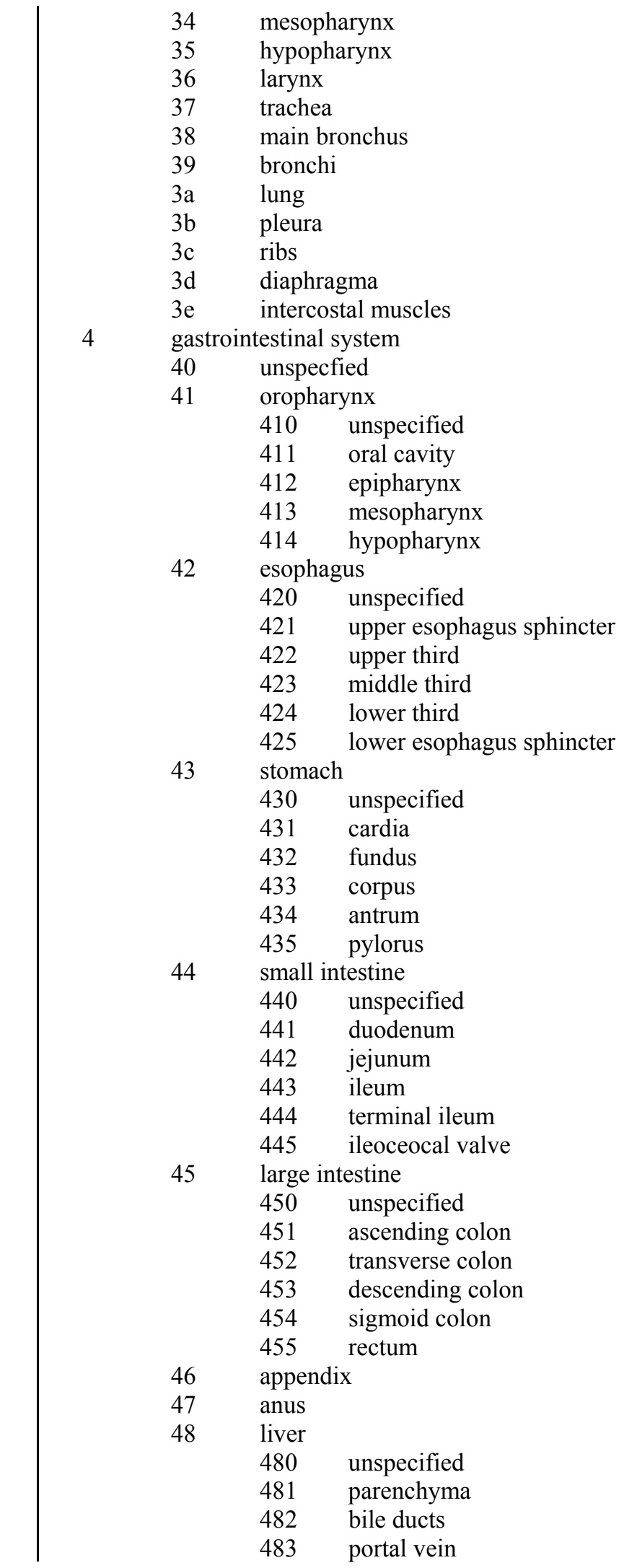


484 arteries

485 veins

49

biliary system

490 unspecified

491 bile ducts

492 gallbladder

4a salivary glands

$4 \mathrm{a} 0$ unspecified

4a1 parotic gland

4a2 sublingual gland

4a3 mandibular gland

4a 4 pancreas

5 uropoietic system

50 unspecified

51 kidney

510 unspecified

511 parenchyma

512 renal pelvis

52 ureter

53 urinary bladder

54 urethra

6

reproductive system

60 unspecified

61 male system

610 unspecified

611 testes

612 deferent duct \&

seminal vesicle

613 prostate gland

614 penis

(incl. corpus cavernosum)

62 female system

620 unspecified

621 ovaries

622 tuba uterina

623 uterus

624 vagina

625 breast

7 musculosceletal system

8 endocrinic system

80 unspecified

81 thyroid gland

82 parathyroid gland

83 adrenal gland

84 pituitary gland

85 hypothalamus

86 ovaries

87 testes

88 pancreas

9

90 unspecified

91 tonsils

92 cervial lymph node

93 axillary lymph node 